\title{
Brain Tumors Diagnostic by Tumor Imaging Agents
}

\author{
Masoud Sadeghzadeh and Fariba Johari Daha \\ Nuclear Science \& Technology Research Institute
}

Iran

\section{Introduction}

Diagnostic nuclear medicine (NM) is among the imaging procedures (together with X-ray, computerized tomography, magnetic resonance, and echography) the clinicians can routinely adopt to image organs or tissues and related disorders. In this chapter, we will describe the role of nuclear medicine imaging in diagnosis of the brain tumors, application of metal complex-carbohydrates for this purpose and review how these imaging approaches can contribute to the diagnosis of disease. The chapter is organized into the following sections:

1. Background

2. Current state of nuclear medicine imaging for brain tumor imaging

3. Introduction another new compounds for brain tumor imaging

\section{Background}

\subsection{Brain tumor}

The brain tumor experience can be a journey into an unknown land filled with uncertainty. Brain tumors occurs when brain cells become abnormal and form cells in an uncontrolled manner. These extra brain cells form into a mass of tissue, also known as a tumor, which can be a classified as benign brain tumor, a mass of extra cells that are harmless and have distinct boundaries, or malignant, a mass of extra cells that are life-threatening and either cancerous or located in a vital brain area. For the most part, malignant brain tumors are indicative of cancer and the American Cancer Society estimate that malignant brain tumors will result in approximately 13,100 deaths (1.4\% of all cancer deaths) in 2004 . Although there are a number of different methods to classify brain tumors, the best way to distinguish between brain tumors is by classifying them as primary brain tumors and secondary brain tumors. A brain tumor diagnosis usually involves several steps, which can include a neurological examination, brain scan(s) and/or a biopsy and nuclear medicine imaging.

\subsection{Brain tumor diagnosis methods}

Identifying a brain tumor usually involves a neurological examination, brain scans, and/or an analysis of the brain tissue. Doctors use the diagnostic information to classify the tumor from the least aggressive (benign) to the most aggressive (malignant). In most cases, a brain tumor is named for the cell type of origin or its location in the brain. Identifying the type of tumor helps doctors determine the most appropriate course of treatment. 
A neurological examination is a series of tests to measure the function of the patient nervous system and physical and mental alertness. If responses to the exam are not normal, the doctor may order a brain scan or refer the patient to a neurologist or neurosurgeon, who will then order a brain scan.

A brain scan is a picture of the internal structures in the brain. A specialized machine takes a scan in much the same way a digital camera takes a photograph. Using computer technology, a scan compiles an image of the brain by photographing it from various angles. Some types of scans use a contrast agent (or contrast dye), which helps the doctor see the difference between normal and abnormal brain tissue. The contrast agent is injected into a vein and flows into brain tissue. Abnormal or diseased brain tissue absorbs more dye than normal healthy tissue. The most common scans used for diagnosis are as follows:

a. MRI (Magnetic Resonance Imaging) is a scanning device that uses magnetic fields and computers to capture images of the brain on film.

b. CT or CAT Scan (Computed Tomography) combines sophisticated x-ray and computer technology. CT can show a combination of soft tissue, bone, and blood vessels. CT images can determine some types of tumors, as well as help detect swelling, bleeding, and bone and tissue calcification. Usually, iodine is the contrast agent used during a CT scan.

c. Nuclear Medicine Imaging provides a picture of the brains activity, rather than its structure. Nuclear medicine imaging non-invasively provides functional information at the molecular and cellular level that contributes to the determination of health status by measuring the uptake and turnover of target-specific radiotracers in tissue. These functional processes include tissue blood flow and metabolism, protein - protein interactions, expression of cell receptors in normal and abnormal cells, cell-cell interactions, neurotransmitter activity, cell trafficking and homing, tissue invasion, and programmed cell death. By providing information on these processes, nuclear medicine imaging offers a broad array of tools for probing normal and disease-related states of tissue function and response to treatment. The addition of anatomic imaging provided by computed tomography (CT) to functional imaging of positron emission tomography (PET) and single photon emission computed tomography (SPECT) has further expanded the utility and accuracy of nuclear medicine imaging (Committee on State of the Science of Nuclear Medicine, 2007).

d. Molecular imaging is expected to have a major impact on the treatment and management of brain tumors.

\subsection{Diagnostic nuclear medicine and imaging techniques}

Nuclear medicine is a highly multi-disciplinary specialty that develops and uses instrumentation and radiopharmaceuticals to study physiological processes and noninvasively diagnose, stage, and treat diseases. A radiopharmaceutical is either a radionuclide alone, such as iodine-131 (table1) or a radionuclide that is attached to a carrier molecule (a drug, protein, or peptide) or particle, which when introduced into the body by injection, swallowing, or inhalation accumulates in the organ or tissue of interest. In a nuclear medicine scan, a radiopharmaceutical is administered to the patient, and an imaging instrument that detects radiation is used to show biochemical changes in the body. Nuclear medicine imaging, in contrast to imaging techniques that mainly show anatomy (e.g., conventional ultrasound, computed tomography [CT], or magnetic resonance imaging [MRI]), can provide important quantitative functional information about normal tissues or 
disease conditions in living subjects. For treatment, highly targeted radiopharmaceuticals may be used to deposit lethal radiation at tumor sites (Committee on State of the Science of Nuclear Medicine, 2007).

\begin{tabular}{|c|c|c|c|c|}
\hline Application & Radionuclide & Half-Life & $\begin{array}{c}\text { Type of Radiation } \\
\text { Emitted }\end{array}$ & $\begin{array}{c}\text { Imaging } \\
\text { Technique Used }\end{array}$ \\
\hline Imaging & Carbon-11 & $20.33 \mathrm{~min}$ & Positron & PET \\
\hline$"$ & Nitrogen-13 & $9.97 \mathrm{~min}$ & $"$ & $"$ \\
\hline$"$ & Oxygen-15 & $2.04 \mathrm{~min}$ & $"$ & $"$ \\
\hline$"$ & Fluorine-18 & $109.75 \mathrm{~min}$ & $"$ & $"$ \\
\hline$"$ & Technetium-99m & 6.02 hours & gamma & SPECT \\
\hline$"$ & Indium-111 & 2.8 days & $"$ & $"$ \\
\hline$"$ & Iodine-123 & 13 hours & $"$ & $"$ \\
\hline$"$ & Thallium-201 & 73 hours & " & \\
\hline Therapy & Iodine-131 & 8 days & beta & \\
\hline$"$ & Yttrium-90 & 2.7 days & $"$ & \\
\hline
\end{tabular}

Table 1. Commonly used radionuclides for imaging and therapy

Diagnostic nuclear medicine (DNM) is among the imaging procedures (together with X-ray, computerized tomography, magnetic resonance, and echography) the clinicians can routinely adopt to image organs or tissues and related disorders. In spite of many advances in diagnosis and therapy, brain tumors still represent a serious challenge for clinicians. Today, contrast-enhanced CT or MRI is the first examination performed in a patient with symptoms typical of brain tumor (i.e. nausea, headache, seizure or focal neurological signs). Both MRI and CT allow exactly localizing brain neoplasia and defining the extension of the tumoral mass to the surrounding normal tissue. Nevertheless, these techniques present some limitations, especially regarding patients' follow-up after treatments when discrimination between tumor recurrence-persistence versus scar tissue is required. In such cases, nuclear functional imaging by PET (Positron Emission Tomography) and SPECT (Single Photon Emission Computed Tomography) was successfully proposed to obtain a metabolic characterization of the morphological lesions detected by MRI or CT scan. By using combined-modality PET/CT and SPECT/CT devices, functional processes can be localized within the body to an anatomically identified or, in some instances, as yet unidentifiable structural alteration. These devices have enhanced the accuracy with which disease can be detected, aided in the determination of the extent and severity of disease, enhanced the accuracy for identifying disease-related risk, and improved the ability to monitor patient response to therapy (Committee on State of the Science of Nuclear Medicine, 2007). In diagnostic nuclear medicine two techniques are used for imaging of tumors:

a. PET (Positron Emission Tomography)

One of the most important uses of Positron Emission Tomography (PET) technology is its use in diagnosing and treating brain tumors by measuring the rate at which a tumor absorbs glucose derivatives or amino acids. In cases where structural change has been detected through MRI or CT, a physician often uses PET imaging to determine the nature of the detected structural changes. The PET scan measures the brains activity and sends this information to a computer, which creates a live image. Doctors use PET scans to see the difference between scar tissue, recurring tumor cells, and necrosis (cells destroyed by 
radiation treatment). PET imaging is a non-invasive diagnostic imaging tool that has an advantage over anatomical imaging tools in that it is a metabolic imaging tool that is able to distinguish between benign and malignant tumors. It is often used to accurately determine the stage of the brain tumor. Brain tumor PET imaging involves the administration of a radioactive tracer that is a combination of a radioisotope (a radioactive compound such as ${ }^{18} \mathrm{~F}$ or ${ }^{11} \mathrm{C}$ whose movements are detectable by a PET scanner) with a natural body compound. When used in brain tumor scanning, the radioactive tracer used in PET is Fluorodeoxyglucose (FDG), which combines the natural body compound glucose with the radioisotope Fluorine-18. This radioactive tracer, or radiopharmaceutical, is used in brain PET imaging as the radioactive compound that it uses has a short half-life that will disappear from the body within hours.

The routinely used PET radiotracer ${ }^{18}$ F-fluorodeoxyglucose (FDG) proved useful to diagnose primary brain tumors and their recurrences; moreover, the entity of FDG uptake was found to correlate with brain tumor histology. Nevertheless, FDG is physiologically taken by normal brain tissue, so this radiotracer is less accurate in detecting very low grade gliomas due to their relatively low target to background uptake ratio. To overcome the drawbacks of FDG, ${ }^{11} \mathrm{C}$-methionine was applied in brain tumors imaging. Its uptake is related to the increased amino acid transport and protein synthesis and it is accumulated in the neoplastic lesion and not in the normal parenchyma. Actually, ${ }^{11} \mathrm{C}$-methionine proved useful to visualize low grade gliomas not detected by FDG (Filippi et al., 2005).

\section{PET and Brain Tumor Follow-Up}

Besides brain tumor staging, Positron Emission Tomography is a valuable tool in brain tumor treatment when it is used as a follow-up to brain tumor treatment. Brain tumors are a difficult to fully treat due to the sensitivity of its location. Therefore, brain tumor recurrence is often possible. Imaging tests such as magnetic resonance imaging (MRI) and computed tomography (CT) are able to be used to detail structural changes, such as tumor formation, in the brain. However, these imaging tests often detect structural changes caused by side effects from previous brain tumor treatments, such as surgery or radiation therapy.

b. SPECT (Single Photon Emission Computed Tomography)

Although PET technology is becoming more and more available in many institutions, its diffusion is still limited and it presents very high cost. Moreover, in the case of ${ }^{11} \mathrm{C}$ methionine an in situ cyclotron should be available due to the short ${ }^{11} \mathrm{C}$ physical half-life. Because the production of ${ }^{18} \mathrm{~F}$ requires a cyclotron and the isotope has a short $(110 \mathrm{~min})$ halflife, its utility is somewhat limited compared to that of single photon emission computed tomography (SPECT) in nuclear medicine. Although conventional scintigraphy (i.e. single photon emission tomography [SPECT] and planar images) presents lower spatial resolution when compared to PET, the high diffusion of Anger camera, the device used to perform conventional scintigraphy, has accounted for the search of $\gamma$-emitting radiotracers as brain tumors imaging agents. In terms of developing novel drugs for use in diagnostic and therapeutic brain tumor in nuclear medicine, it would be of great interest to label sugars (such as glucose and analogue derivatives) and amino acids with the transition metal isotopes (such as ${ }^{99} \mathrm{mTc}, 186 / 188 \mathrm{Re},{ }^{201} \mathrm{Tl},{ }^{123} \mathrm{I}$, etc.) to substitute the expensive but readily used 18F-labeled 2-deoxyglucose (18F-FDG) for localization of tumor and metastatic tissue. 201Tallium was firstly used to detect cerebral neoplasia showing high sensitivity but relatively low specificity mainly due to its physical characteristics suboptimal for imaging with Anger camera (Filippi et al., 2005). 


\section{Current state of diagnostic nuclear medicine for brain tumor imaging}

\subsection{Anatomic imaging procedures (CT scan and MRI)}

Anatomic imaging procedures (computed tomography [CT] and magnetic resonance imaging [MRI]) have become essential tools for brain tumor assessment. Serial MRI is routinely performed in these patients after primary treatment to detect tumor recurrence. However, conventional contrast-enhanced CT scans or MRI cannot reliably distinguish radiation necrosis from recurrent tumor. Both entities can cause extensive edema and blood-brain barrier disruption that result in mass effect and abnormal contrast enhancement.2,3 Radiation-induced necrosis often occurs within 2 years after radiation therapy, the same time frame during which tumor recurrence is most frequent. Differentiation between tumor progression and radiation necrosis carries obvious prognostic and therapeutic implications. To overcome this problem, several functional and physiological imaging techniques, such as MR spectroscopy (MRS), perfusion-weighted MRI, positron emission tomography (PET), and thallium-201 single-photon emission computed tomography (201Tl-SPECT) have been examined for clinical use (Tie et al., 2008).

\subsection{Functional imaging procedures (PET and SPECT)}

Using anatomical imaging modalities, such as computed tomography (CT) or magnetic resonance imaging (MRI), it is difficult to differentiate tumor recurrence from necrosis induced by radiotherapy or other treatments. Functional imaging of the brain, in the form single photon emission computed tomography (SPECT) utilizing various "tumor seeking" tracers, is the most commonly performed investigation to differentiate recurrence from necrosis (Barai et al., 2005). Functional imaging procedures (positron emission tomography [PET] and singe-photon emission computed tomography [SPECT]) can provide additional information useful during the diagnostic workup to determine the degree of malignancy and as a substitute or guide for biopsy. After surgery and/or radiotherapy, nuclear medicine examinations are essential to assess persistence of tumor, to differentiate recurrence from radiation necrosis and gliosis, and to monitor the disease. The combination of functional images with anatomic ones is of the utmost importance for a full evaluation of these patients, which can be obtained by means of imaging fusion. Brain imaging is performed using radiopharmaceuticals by single photon emission computed tomography (SPECT) and positron emission tomography (PET). SPECT and PET radiopharmaceuticals are classified according to blood-brain-barrier permeability, cerebral perfusion and metabolism receptor-binding, and antigen-antibody binding.

\subsubsection{PET radiopharmaceuticals}

PET radiopharmaceuticals for brain imaging are commonly labeled with positron-emitters such as ${ }^{11} \mathrm{C},{ }^{13} \mathrm{~N},{ }^{15} \mathrm{O}$, and ${ }^{18} \mathrm{~F}$, although other radionuclides such as ${ }^{82} \mathrm{Rb},{ }^{62} \mathrm{Cu}$ and ${ }^{68} \mathrm{Ga}$ also were used. The brain uptake of $\left[{ }^{13} \mathrm{~N}\right]$ glutamate, $\left[{ }^{68} \mathrm{Ga}\right] \mathrm{EDTA}$ and $\left[{ }^{82} \mathrm{Rb}\right] \mathrm{RbCl}$ depends on the BBB permeability, but these are rarely used for brain imaging. Several cerebral perfusion agents have been introduced, of which $\left[{ }^{15} \mathrm{O}\right]$ water, $\left[{ }^{13} \mathrm{~N}\right]$ ammonia, and $\left[{ }^{15} \mathrm{O}\right]$ butanol have been used more frequently. Regional $\mathrm{CBF}$ has been quantitated by using these tracers in normal and different cerebral disease states. Other perfusion agents include $\left[{ }^{15} \mathrm{O}\right] \mathrm{O}_{2}$, $\left[{ }^{11} \mathrm{C}\right] \mathrm{CO},\left[{ }^{11} \mathrm{C}\left[\mathrm{CO}_{2},\left[{ }^{18} \mathrm{~F}\right]\right.\right.$ fluoromethane, $\left[{ }^{15} \mathrm{O}\right] \mathrm{O}_{2},\left[{ }^{11} \mathrm{C}\right]$ butanol, and $\left[{ }^{62} \mathrm{Cu}\right] \mathrm{PTSM}$. Among the PET cerebral metabolic agents, $\left.{ }^{18} \mathrm{~F}\right]$ fluoro-deoxyglucose (FDG) is most commonly used to detect metabolic abnormalities in the brain. Various brain tumors have been graded by 
[18F]FDG PET. This technique was used to detect epileptic foci by showing increased uptake in the foci during the ictal period and decreased uptake in the interictal period. Differentiation between recurrent tumors and radiation necrosis and the detection of Alzheimer's disease have been made successfully by [18F]FDG PET. Other PET metabolic agents such as $\left[{ }^{11} \mathrm{C}\right]$ deoxyglucose, and $\left.{ }^{111} \mathrm{C}\right]$ methylmethionine have drawn attention in the detection of brain tumors. [18F]fluorodopa is a cerebral neurotransmitter agent, which has been found very useful in the detection of Parkinson disease that shows reduced uptake of the tracer in the striatum of the brain. The distribution of different receptors in the brain and their alteration in different disease states are assessed by using different receptor-binding PET radiotracers such as $\left[{ }^{11} \mathrm{C}\right]$ methylspiperone, [18 $\left.\mathrm{F}\right]$ fluoroethylspiperone, [18fluoropropylspiperone, [11C]carfentanil, [11C]diprenorphine, [11C]raclopride, [11C]flumasenil, and so on (Saha et al., 1994).

\subsubsection{SPECT radiopharmaceuticals}

Despite the fast-growing diffusion of PET, in most cases of brain tumors, SPECT studies are adequate and provide results that parallel those obtained with PET. The main limitation of SPECT imaging with brain tumor-seeking radiopharmaceuticals is the lack of precise anatomic details; this drawback is overcome by the fusion with morphological studies that provide an anatomic map to scintigraphic data. In the past, software-based fusion of independently performed SPECT and CT or MRI demonstrated usefulness for brain tumor assessment, but this process is often time consuming and not practical for everyday nuclear medicine studies. The recent development of dual-modality integrated imaging systems, which allow the acquisition of SPECT and CT images in the same scanning session, and their coregistration by means of the hardware, has facilitated this process. In SPECT studies of brain tumors with various radiopharmaceuticals, fused images are helpful in providing the precise localization of neoplastic lesions, and in excluding the disease in sites of physiologic tracer uptake. This information is useful for optimizing diagnosis, therapy monitoring, and radiotherapy treatment planning, with a positive impact on patient management. Imaging Fusion of SPECT Studies (Schillaci et al., 2007):

1. $201 \mathrm{Tl}$

2. $99 \mathrm{mTc}$ Sestamibi

3. $99 \mathrm{mTc}$ Tetrofosmin

4. 123I-Alpha-Methyl-Tyrosine

5. 111In-Pentetreotide

6. Labeled Leukocytes

a. Clinical used radiopharmaceuticals

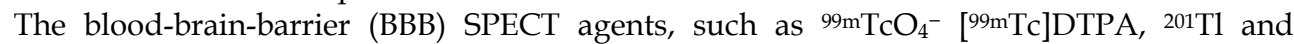
$\left[{ }^{67} \mathrm{Ga}\right]$ citrate are excluded by normal brain cells, but enter into tumor cells because of altered BBB. These agents were used in the earlier period for the detection of brain tumors. SPECT perfusion agents such as [123I]IMP, [99mTc]HMPAO, [99mTc]ECD are lipophilic agents and therefore, diffuse into the normal brain. These tracers have been successfully used to detect various cerebrovascular diseases such as stroke, Parkinson disease, Huntington's disease, epilepsy, dementia, and psychiatric disorders. Xenon-133 and radiolabeled microspheres have been used for the measurement of cerebral blood flow (CBF). Important receptorbinding SPECT radiopharmaceuticals include [123I]QNE, [123I]IBZM, and [123I]iomazenil. These tracers bind to specific receptors in the brain, thus displaying their distribution in 
various receptor-related cerebral diseases. Radioiodinated monoclonal antibodies were used for the detection of brain tumors.

A variety of radiolabeled amino acids have been developed as potential tumor imaging agents for positron emission tomography (PET) and single photon emission computerized tomography (SPECT). Many radiolabeled amino acids developed for tumor imaging enter cells via the A-type (sodium dependent) and/or the L-type (sodium independent) transport systems, which are upregulated in many neoplasms. The L-type substrate [123I]IMT represents the most widely used amino acid for tumor imaging with SPECT (Yu et al., 2008). MIBI and Tetrofosmin are technetium labeled compounds which were firstly introduced as imaging agent for myocardial scintigraphy. These two radiopharmaceuticals are passively accumulated in cells characterized by high metabolic activity so they have been successfully used also in oncology (i.e. lung, parathyroid, breast cancer). Regarding brain tumors imaging, MIBI was widely applied in diagnosis and follow-up, while few experiences were performed using Tetrofosmin. MIBI was proved useful to detect intracranial tumors before therapy with higher sensitivity and specificity for gliomas than for the other histologies (i.e. lymphomas). Moreover, the entity of MIBI uptake was found to be related to the grade of malignancy of gliomas. In patients' follow-up, MIBI scintigraphy showed great diagnostic accuracy to discriminate tumor recurrence versus scar tissue also when compared to CT scan. As MIBI is a substrate of Pgp, it was proposed to diagnose chemoresistance in brain tumors patients. Nevertheless, the preliminary experiences suggest that Pgp expression is inversely related to the grade of malignancy of gliomas so that Pgp seems not to be the main cause of chemoresistance in these tumors. Tetrofosmin scintigraphy presents similar characterisitics in sensitivity and specificity as MIBI, so it may represent a suitable tool to image brain tumors (Filippi et al., 2005).

b. New research radiopharmaceuticals

Flourine-18 $\left({ }^{18} \mathrm{~F}\right)$ fluorodeoxyglucose (FDG) has been used to measure normal tissue and tumor glucose utilization rates. Although metabolic tumor imaging with [18F]-FDG has been studied for more than two decades, the use of this examination in clinical practice is still limited by factors such as difficult access, limited availability, and high cost. In addition, positron emission tomography (PET) radiosynthesis must be performed rapidly because the half-life of ${ }^{18} \mathrm{~F}$ is only $109 \mathrm{~min}$. Thus, it would be very desirable to develop less costly imaging agents based on $\gamma$-emitter isotopes, especially for developing countries, where single photon emission computed tomography (SPECT) is still dominant. Technetium-99m $\left({ }^{99 \mathrm{mTc})}\right.$ has been mostly used for radiopharmaceuticals labeling due to its suitable physical and chemical characteristics and inexpensive isotope cost. Organometallic metal cores often exhibit advantages in terms of kinetic inertness, stability, and size and thus could lead to the development of more efficient and stable compounds compared to classical inorganic complexes. We elected to use the versatile low valent fac- $\left[{ }^{99 \mathrm{~m} T c}(\mathrm{I})-(\mathrm{CO})_{3}\right]$ core, which its chemistry has recently been pioneered by Alberto and co-workers. The facially coordinated carbonyl ligands stabilize the $\mathrm{Tc}^{+1}$ oxidation state, obviating the elaborate, often macrocyclic, polydentate structures required to stabilize other intermediate oxidation states of Tc. In neutral complexes with simple $\mathrm{N}, \mathrm{O}$ donors the fac-[99m $\left.\mathrm{Tc}(\mathrm{I})-(\mathrm{CO})_{3}\right]$ core possesses intermediate lipophilicity, an advantage in living systems. Lots of $99 \mathrm{mTc}$-labeled glucose derivatives have been synthesized in order to develop one subrogate in SPECT for [ $\left.{ }^{18} \mathrm{~F}\right]-\mathrm{FDG}$ in PET recently. Developed by Yang, 99mTc-labeled ethylenedicysteine-deoxyglucose (ECDG) showed similarities with $[18 \mathrm{~F}]-\mathrm{FDG}$ in tumor uptake. This suggests that there is 
feasibility for ${ }^{99 \mathrm{mTc}} \mathrm{c}$ labeled deoxyglucose as a metabolic tumor imaging agent. However, [99mTc]-EC-DG still has some drawbacks such as slow cleanup from the blood, which would cause high background activity; and large molecular weight, which would limit its penetration through blood-brain barrier (BBB). Thus, it would be desirable to develop a smaller 99mTc based deoxyglucose derivative with rapid blood clearance and still maintaining its high tumor uptake. In terms of developing novel drugs for use in diagnostic nuclear medicine, it would be of great interest to label glucosamine derivatives with ${ }^{99 \mathrm{mTc}}$ to substitute the expensive but readily used ${ }^{18 F}$-labeled 2-deoxy glucose (18F-FDG) for

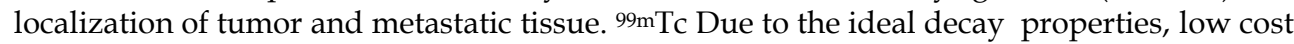
production is used the most frequently in nuclear medicine today. Reported by Sadeghzadeh, three glucosamine derivatives showed negligible to moderate brain uptake similar to $99 \mathrm{mTc}-\mathrm{EC}$ (Johari et al., 2007). Compounds (1) and (2) were prepared in one step by reaction of glucosamine, ethylcholoroformate and 3-chloro-1,2-propandiol as starting materials (Scheme1).
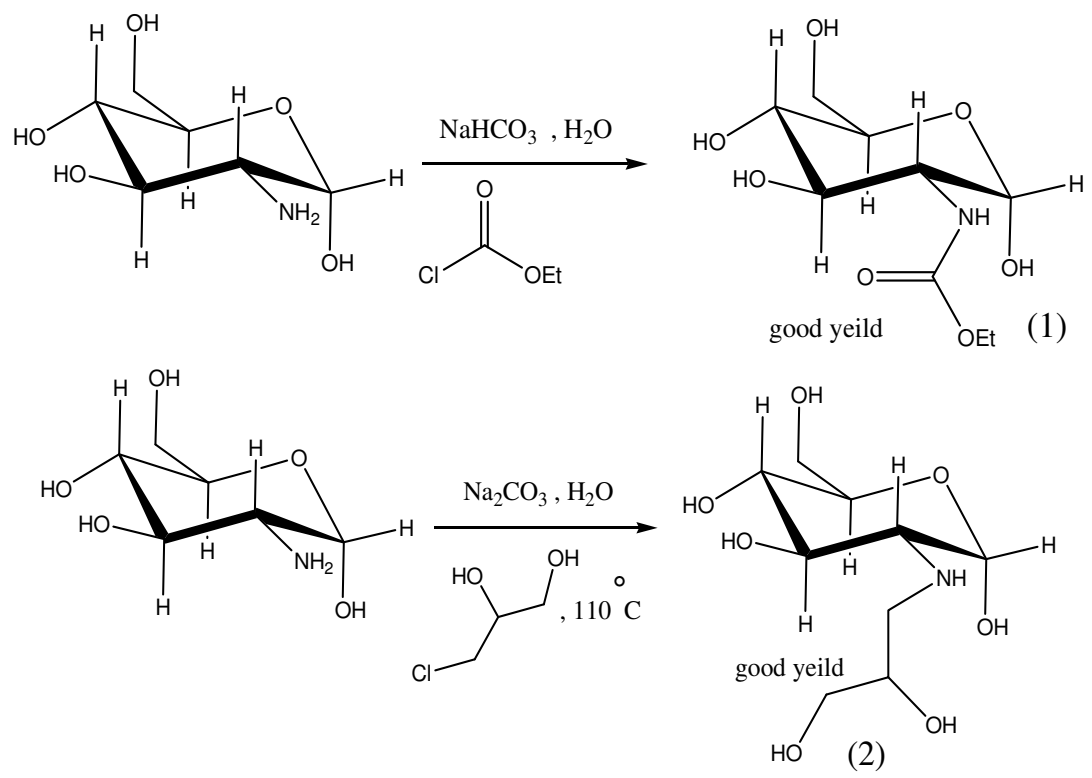

Scheme1. Syntesis of two glucosamine derivatives

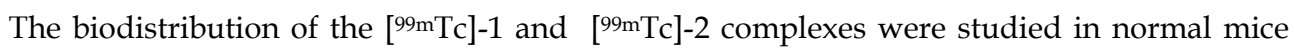
(body mass $25-35 \mathrm{~g}$ ) at $30 \mathrm{~min}$ and $1 \mathrm{~h}$ post-injection, according to a published procedure. These complexes showed moderate brain uptake $(0.613 \% \pm 0.03$ ID) and $(0.512 \% \pm 0.03$ ID) at 30 min post-injection, an efficient clearance from the blood, a rapid excretion to the urine and a low retention in the liver and kidneys. Planar SPECT imaging of the compound1 showed moderate uptake brain in mice after 2 hours administration (Figure 1).

At a same work, the proligand 3 is prepared by thioglycolic acid as a starting material

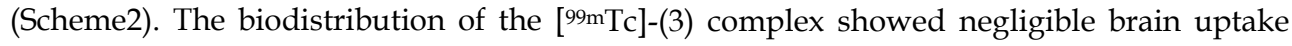
$(0.13 \% \pm 0.03 \mathrm{ID})$ at $30 \mathrm{~min}$ post-injection (Johari et al., 2007). 


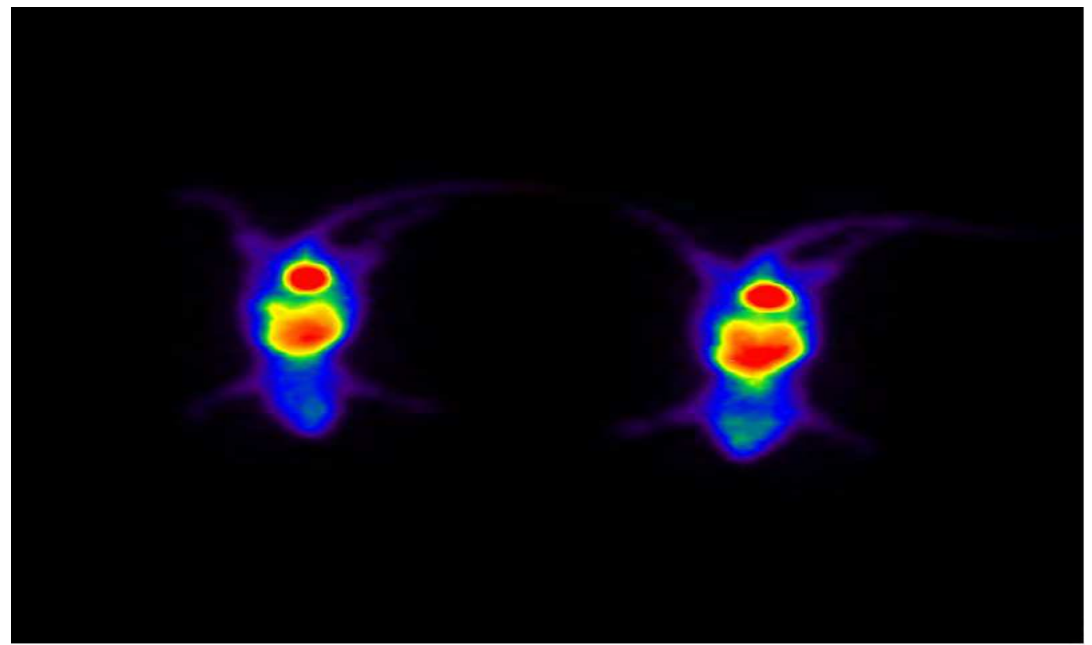

Fig. 1. Planar SPECT imaging of the compound1 after 2 hours administration.

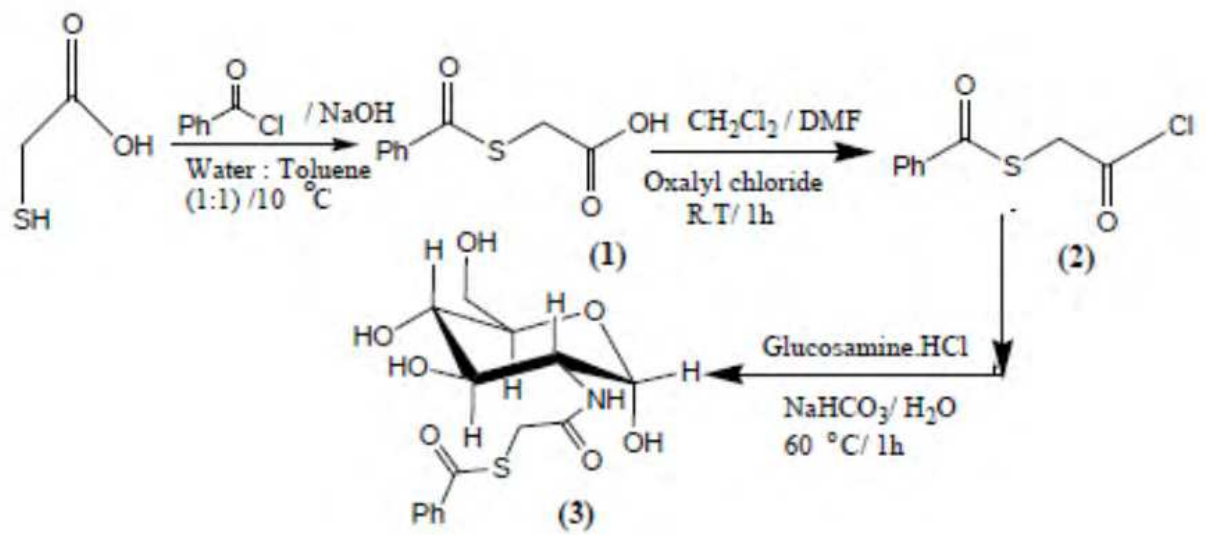

Scheme 2. Synthesis of 2-Amino-2-Deoxy(S-Benzoylthioacetyl)-D-Glucose (3)

\section{New imaging agents for brain tumor diagnosis}

\subsection{Molecular imaging (Phosphatidylserine (PS) as a marker for brain tumor)}

Noninvasive molecular-imaging technologies are playing a keyrole in drug discovery, development and delivery. Positron Emission Tomography (PET) is such a molecular imaging technology and a powerful tool for the observation of various diseases. Anionic phospholipids are largely absent from the surface of resting mammalian cells under normal conditions. PS, which is the most abundant anionic phospholipid of the plasma membrane, is tightly segregated to the internal leaflet of the plasma membrane in most cell types. 
- $\quad$ Anionic phospholipid-targeting agent

PSVueTM reagents are a family of fluorescent probes containing a bis ( $\mathrm{Zn}^{2+}$-dipicolylamine) group (Zn-DPA), a motif that has been found to bind with high affinity to surfaces enriched with anionic phospholipids, especially phosphatidylserine (PS) exposed on cell membranes (Figure 2). The fluorescent part of the probe is a reporter element that provides a means of detecting the probe once it is bound to the membrane of interest. Key features of PSVueTM Probes are following: (I) Bind to a tumor cells which have negatively charged phospholipids exposed on their membranes. (II) Available in a range of detection wavelengths from longUV to near infrared. (III) Suitable for in vitro and in vivo use. (IV) Suitable for highthroughput screening assays. PSVueTM binds to the same PS site as annexin-V (Qi et al., 2010). Location of PSVue794 in tumor bearing mice revealed by fluorescence imaging. Photon emission from tumors or tissues was detected, digitized and electronically displayed as a pseudocolor overlay onto a gray scale animal image. Luminescence and fluorescence imaging of excised brains was also performed using the IVIS-200 system (Figure 3).

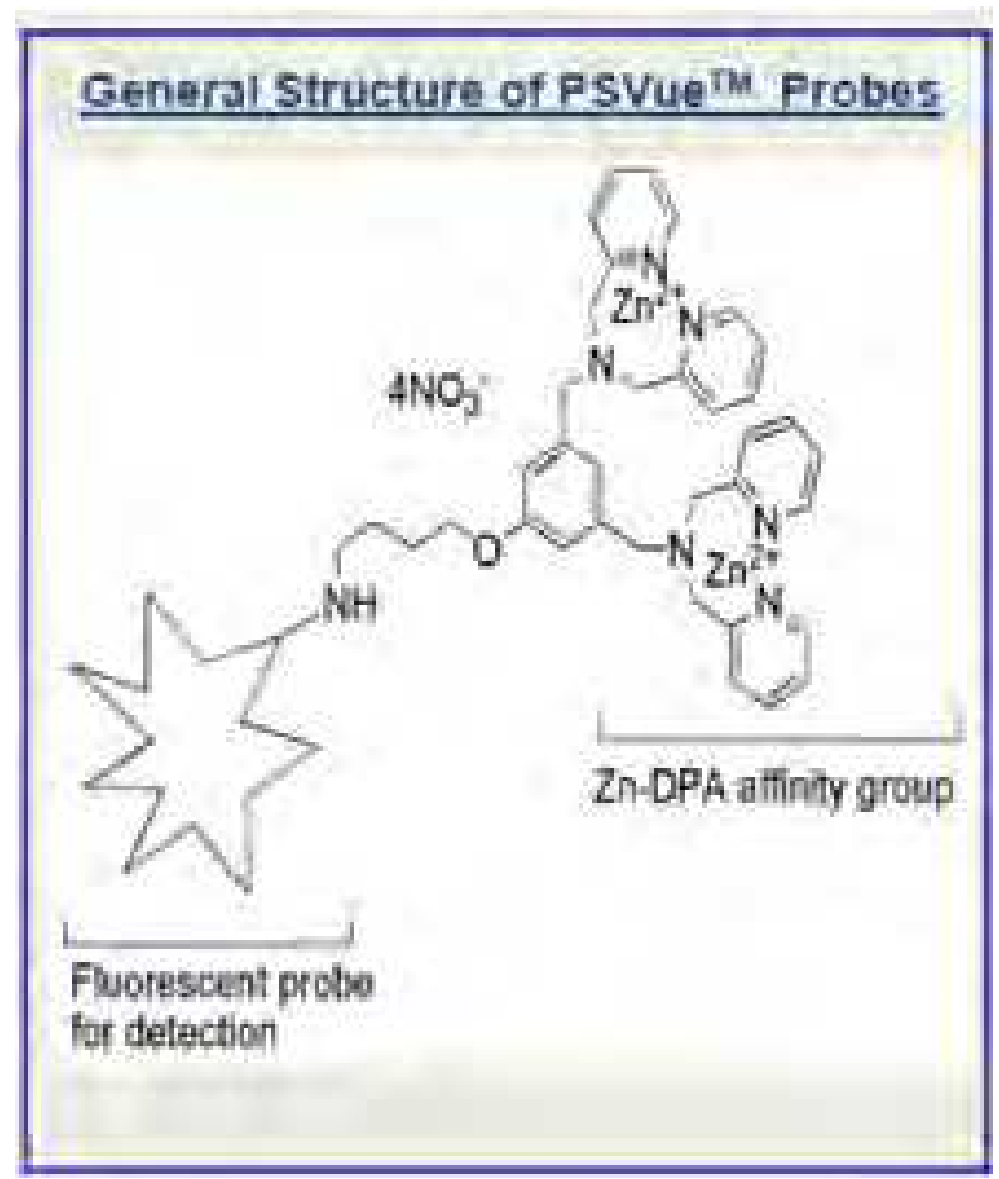

Fig. 2. General structure of PSVue ${ }^{\mathrm{TM}}$ Probes 


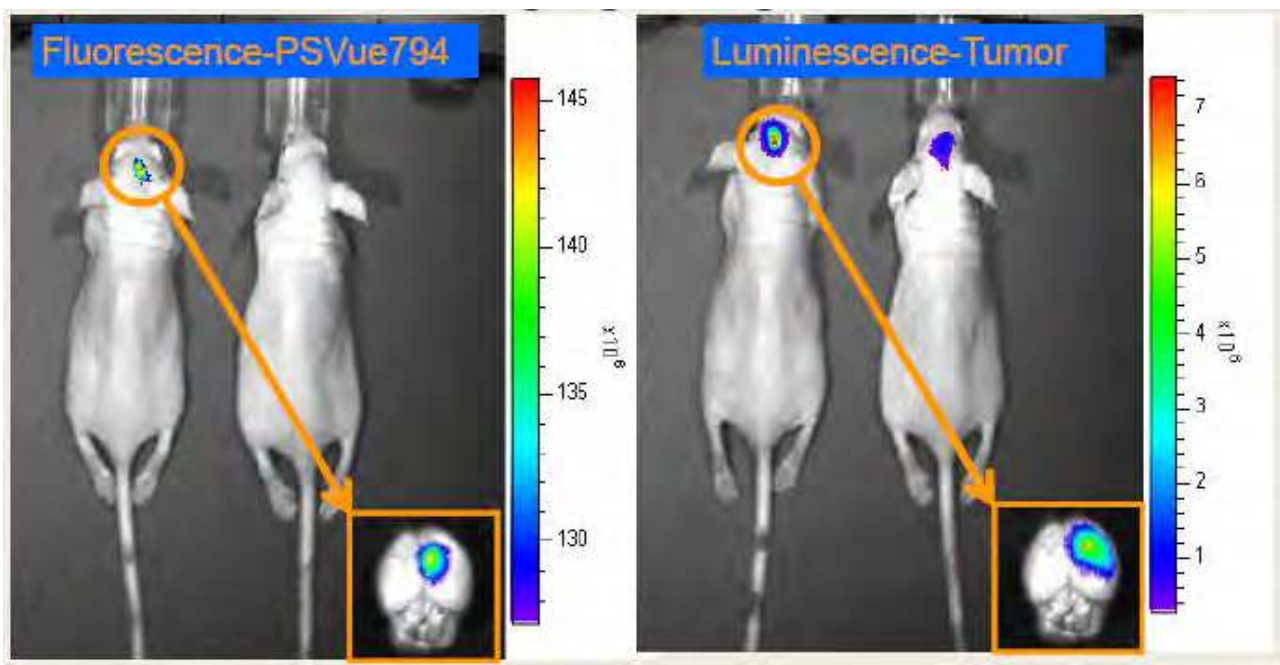

Fig. 3. Brian-Tumor Imaging using PSVue794

Dose-dependent imaging of brain tumor by tail vein injection of PSVue794 for $24 \mathrm{~h}$ (Figure 4). Left panel: fluorescence imaging with $1 \mathrm{sec}$ imaging time, 745/840 filter set. Right panel Luminescnece imaging for tumors with $10 \mathrm{sec}$ imaging time.

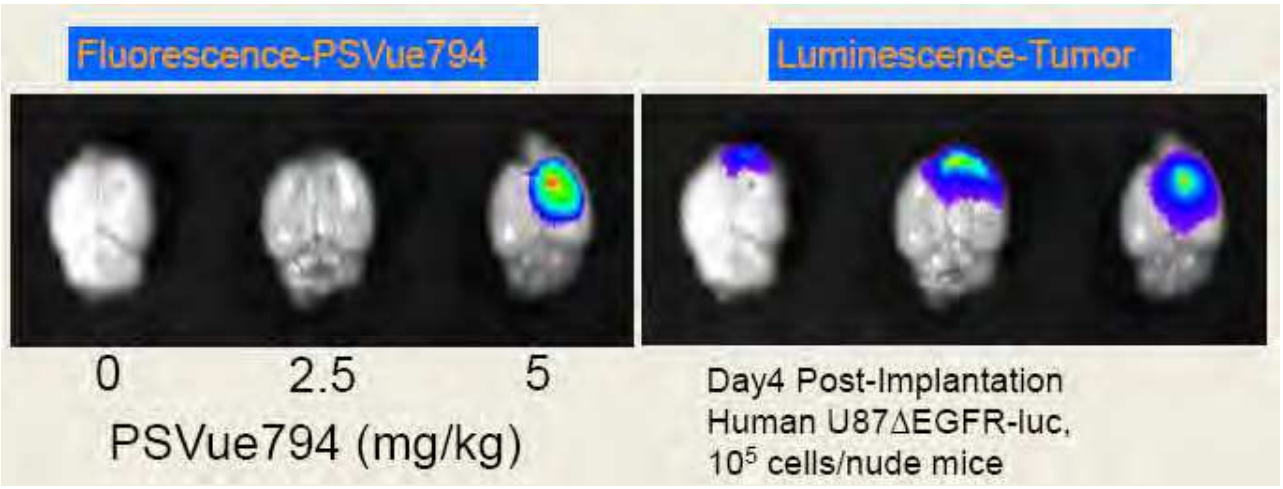

Fig. 4. Dose-Dependent Brain Tumor Imaging

\subsection{Nanomedicine techniques}

The field of nanomedicine is expected to have a major impact on the treatment and management of brain tumors. Over the past decade, significant efforts have been made in using nanoparticles for diagnosis and treatment of brain tumors. One class of nanoparticles, liposomes, have received considerable attention for use as nanocarriers for delivery of therapeutics and contrast agents. 


\section{References}

Barai S., Rajkamal, Bandopadhayaya G. P., Pant G. S., Haloi A. K., Malhotra A. \& Dhanpathi H. 2005. Thallium-201 versus 99mTc-glucoheptonate SPECT for evaluation of recurrent brain tumors: a within-subject comparison with pathological correlation, Journal of Clinical Neuroscience, Vol. 12, No. 1, pp. 27-31.

Committee on State of the Science of Nuclear Medicine, National Research Council. 2007. Advancing Nuclear Medicine Through Innovation, THE NATIONAL ACADEMIES PRESS, ISBN: 0-309-11068-8, U.S.A., Washington D.C.

Filippi L., Santoni R., Manni C., Danieli R., Floris R. \& Schillaci O. 2005. Imaging Primary Brain Tumors by Single-Photon Emission Computerized Tomography (SPECT) with Technetium-99m Sestamibi (MIBI) and Tetrofosmin, Current Medical Imaging Reviews, Vol. 1, No. 1, pp. 61-66, 1573-4056/05.

Johari D. F., Sadeghzadeh M., Charkhlooie A., Ebrahimabadi K. H. \& Saidi M. R. 2007. An Improved Synthesis and Preliminary Biodistribution Study of a Technetium-99mlabeled 2-amino-2-deoxy(thioacetyl)-D-glucose Complex ([99mTc]-TA-DG) As a Tumor Imaging Agent. Iran J Nucl Med, Vol. 15, No. 2, September 2007, pp. 43-48.

Qi X., Chu Z., Kaur B., Chiocca A., Gray B. D., Smith B. D. \& Pak K. Y. 2010. Anionic Phospholipid-Targeting Agent for Brain Tumor Imaging, 2010 World Molecular Imaging Congress, Kyoto, Japan, September 8-11.

Schillaci O., Filippi L., Manni C. \& Santoni R. Seminars in Nuclear Medicine, Vol. 37(1), January 2007, 34-47.

Saha G. B., MacIntyre W. J. \& Go R. T. Seminars in Nuclear Medicine, Vol. 4(4), October 1994, 324-349.

Tie J., Gunawardana D. H. \& Rosenthal M. A. 2008. Differentiation of tumor recurrence from radiation necrosis in high-grade gliomas using ${ }^{201} \mathrm{Tl}-\mathrm{SPECT}$, Journal of Clinical Neuroscience, Vol. 15, pp. 1327-1334.

Yu W., Williams L., Malveaux E., Camp V. M., Olsonb J. J. \& Goodman M. M. 2008. Synthesis and evaluation of [123I] labeled iodovinyl amino acids syn-, anti-1-amino3-[2-iodoethenyl]-cyclobutane-1-carboxylic acid, and 1-amino-3-iodomethylenecyclobutane-1-carboxylic acid as potential SPECT brain tumor imaging agents, Bioorganic \& Medicinal Chemistry Letters, Vol. 18, pp. 1264 -1268. 


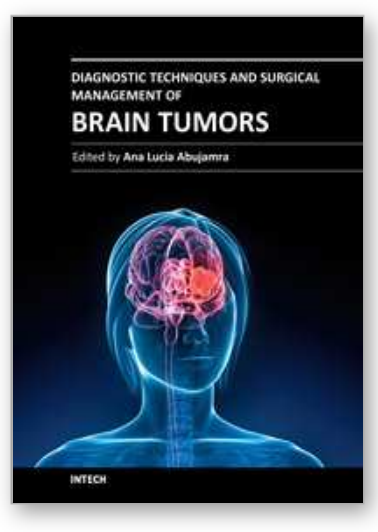

\author{
Diagnostic Techniques and Surgical Management of Brain Tumors \\ Edited by Dr. Ana Lucia Abujamra
}

ISBN 978-953-307-589-1

Hard cover, 544 pages

Publisher InTech

Published online 22, September, 2011

Published in print edition September, 2011

The focus of the book Diagnostic Techniques and Surgical Management of Brain Tumors is on describing the established and newly-arising techniques to diagnose central nervous system tumors, with a special focus on neuroimaging, followed by a discussion on the neurosurgical guidelines and techniques to manage and treat this disease. Each chapter in the Diagnostic Techniques and Surgical Management of Brain Tumors is authored by international experts with extensive experience in the areas covered.

\title{
How to reference
}

In order to correctly reference this scholarly work, feel free to copy and paste the following:

Masoud Sadeghzadeh and Fariba Johari Daha (2011). Brain Tumors Diagnostic by Tumor Imaging Agents, Diagnostic Techniques and Surgical Management of Brain Tumors, Dr. Ana Lucia Abujamra (Ed.), ISBN: 978953-307-589-1, InTech, Available from: http://www.intechopen.com/books/diagnostic-techniques-and-surgicalmanagement-of-brain-tumors/brain-tumors-diagnostic-by-tumor-imaging-agents

\section{INTECH}

open science | open minds

\section{InTech Europe}

University Campus STeP Ri

Slavka Krautzeka 83/A

51000 Rijeka, Croatia

Phone: +385 (51) 770447

Fax: +385 (51) 686166

www.intechopen.com

\section{InTech China}

Unit 405, Office Block, Hotel Equatorial Shanghai

No.65, Yan An Road (West), Shanghai, 200040, China

中国上海市延安西路65号上海国际贵都大饭店办公楼 405 单元

Phone: +86-21-62489820

Fax: $+86-21-62489821$ 
(C) 2011 The Author(s). Licensee IntechOpen. This chapter is distributed under the terms of the Creative Commons Attribution-NonCommercialShareAlike-3.0 License, which permits use, distribution and reproduction for non-commercial purposes, provided the original is properly cited and derivative works building on this content are distributed under the same license. 